\title{
The Hölder Continuity of Solutions to Generalized Vector Equilibrium Problems ${ }^{1}$
}

\author{
S. J. $\mathrm{LI}^{2}$, X. B. LI, L. N. WANG \\ College of Mathematics and Science, \\ Chongqing University, Chongqing, 400030, China \\ E-mails: lisj@cqu.edu.cn, xiaobinglicq@126.com, lnw419@163.com \\ K. L. TEO \\ Department of Mathematics and Statistics, \\ Curtin University of Technology, G.P.O. Box U1987, Perth, WA 6845, Australia \\ E-mail: K.L.Teo@curtin.edu.au
}

November 18, 2008

\begin{abstract}
In this paper, by using a weaker assumption, we discuss the Hölder continuity of solution maps for two cases of parametric generalized vector equilibrium problems under the case that the solution map is a general set-valued one, but not a single-valued one. These results extend the recent ones in the literature. Several examples are given for the illustration of our results.
\end{abstract}

Keywords: Generalized vector equilibrium problems; Hölder continuity; Hausdorff distance; Metric spaces

\section{Introduction}

It is well known that the vector equilibrium problem (VEP, in short) provides a unified model of several classes of problems, for example, vector variational inequality problems, vector complementarity problems, vector optimization problems and vector saddle point problems. Many authors (see $[8,9,12,17,18]$ ) have intensively studied different types of vector equilibrium problems.

\footnotetext{
${ }^{1}$ This research was partially supported by the National Natural Science Foundation of China (Grant numbers: 10871216 and 60574073) and Natural Science Foundation Project of CQ CSTC (Grant number: 2007BB6117).

${ }^{2}$ Corresponding author
} 
The stability analysis of solution maps for equilibrium problems is an important topic in optimization theory and applications. There have been many papers to discuss the semicontinuity of solution maps (see $[1,4,6,7,13,15,16]$ ). However, there are only a few results concerning the Hölder continuity of solution maps for the perturbed variational inequality and equilibrium problems. In [22], by the orthogonal metric projection and the linearity canonical pair $\langle\cdot, \cdot\rangle$ in Hilbert spaces, Yen proved that the solutions of a classical perturbed variational inequalities is unique in some neighborhood, and then obtained Hölder continuity of the single valued solution map in the neighborhood. Based on the concepts of strong monotonicity and Hölder continuity, Ait Mansour and Riahi [19] obtained the uniqueness and Hölder continuity of the solutions in some neighborhood for a parametric scalar equilibrium problem, which can be viewed as an extension and the generalization of the well-known results on variational inequalities in [22]. In [11], by virtue of the strongly pseudomonotone and Hölder continuous concepts, Bianchi and Pini proved the solutions of a parametric scalar equilibrium problems also is unique in some neighborhood and then obtained the Hölder continuity of the unique solution in the neighborhood. In [2], Anh and Khanh generalized the main results of [11] to vector case and obtained uniqueness and Hölder continuity of the solutions in some neighborhood for two classes of perturbed generalized vector equilibrium problems. In [3], Anh and Khanh further discussed uniqueness and Hölder continuity of the solutions for the perturbed generalized vector equilibrium problems, which remarkably improve the results in [11] and [2], and which become properly stronger than the the result of [22] when applied to variational inequalities. In [5], Anh and Khanh extended the results of $[2,3,19]$ to the case of the perturbed generalized vector quasiequilibrium problems, and obtained the Hölder continuity and uniqueness of the solutions for perturbed generalized vector quasiequilibrium problems. Obviously, all these results for Hölder continuity of the solutions in a neighborhood are obtained under the case that the solutions is unique in the neighborhood under very strong conditions.

For general perturbed (vector) equilibrium problems, it is well known that a solution map is, in general, a set-valued one, but not a single-valued one. Until now there is no paper to study Hölder continuity of the solution map when the solution map is a general set-valued one for general perturbed (vector) equilibrium problems. Naturally, there is a need to study the properties of the solution map in this case. So, in this paper, our aims are to investigate the Hölder continuity of the solution maps, which are not single-valued ones, for two classes of parametric generalized vector equilibrium problems in general 
metric spaces. We show the sufficient condition which guarantees the Hölder continuity of the solution maps for the two classes of parametric generalized vector equilibrium problems, and give some examples to illustrate that our main results are different from the corresponding ones in $[2,11]$.

The rest of the paper is organized as follows. In Section 2, we introduce two classes of parametric generalized vector equilibrium problems, and recall some definitions and their properties. In Sections 3 and 4, we discuss the Hölder continuity of the solution maps for the two cases of parametric generalized vector equilibrium problems respectively, and provide remarks and examples to compare with recent results, respectively.

\section{Preliminary Results}

Throughout this paper, unless otherwise specified, let $X, Y, \Lambda$ and $M$ be four metric spaces, and let $E \subset X$ be a nonempty subset. We also assume that $C \subset Y$ is a nonempty closed convex cone of $Y$ with $\operatorname{int} C \neq \emptyset$, where $\operatorname{int} C$ denotes the interior of $C$.

Consider the following two classes of generalized vector equilibrium problems of finding $\bar{x} \in E$ such that

$$
\text { (GVEP1) } \quad F(\bar{x}, y) \nsubseteq-\operatorname{int} C, \quad \forall y \in E ;
$$

and of finding $\bar{x} \in E$ such that

$$
(\text { GVEP2) } \quad F(\bar{x}, y) \subseteq Y \backslash-\operatorname{int} C, \quad \forall y \in E,
$$

where $E$ is a subset of $X$ and $F: E \times E \rightarrow 2^{Y}$ is a set-valued map.

When the set $E$ and the set-valued map $F$ are, respectively, perturbed by the parameters $\lambda \in \Lambda$ and $\mu \in M$, we define the two classes parametric generalized vector equilibrium problems of finding $\bar{x} \in E(\lambda)$ such that

$$
(\mathrm{PGVEP} 1) \quad F(\bar{x}, y, \mu) \not \subset-\operatorname{int} C, \quad \forall y \in E(\lambda),
$$

and of finding $\bar{x} \in E(\lambda)$ such that

$$
(\mathrm{PGVEP} 2) \quad F(\bar{x}, y, \mu) \subseteq Y \backslash-\operatorname{int} C, \quad \forall y \in E(\lambda),
$$

where $E: \Lambda \rightarrow E$ and $F: E \times E \times M \rightarrow 2^{Y}$ are two set-valued maps. 
For each $\lambda \in \Lambda, \mu \in M$, by $S_{1}(\lambda, \mu)$ we denote the solution map of (PGVEP1), i.e.,

$$
S_{1}(\lambda, \mu)=\{x \in E(\lambda): F(x, y, \mu) \nsubseteq-\operatorname{int} C, \forall y \in E(\lambda)\},
$$

and for each $\lambda \in \Lambda, \mu \in M$, by $S_{2}(\lambda, \mu)$ we denote the solution map of (PGVEP2), i.e.,

$$
S_{2}(\lambda, \mu)=\{x \in E(\lambda): F(\bar{x}, y, \mu) \subseteq Y \backslash-\operatorname{int} C, \forall y \in E(\lambda)\}
$$

Throughout this paper, we always assume that $S_{1}(\lambda, \mu) \neq \emptyset$ and $S_{2}(\lambda, \mu) \neq \emptyset$ in a neighborhood of some point $(\bar{\lambda}, \bar{\mu})$.

Now we recall some basic definitions and their properties which are needed in this paper. In the sequel, $B(0, \delta)$ denotes the closed ball with center $0 \in X$ and radius $\delta \geq 0$, and $d(\cdot, \cdot)$ denotes the distance in metric spaces.

Definition 2.1 [10] Let $X$ and $Y$ be two topological spaces, and $G: X \rightarrow 2^{Y}$ be a set-valued map.

(i) The map $F$ is said to be upper semicontinuous at $x_{0} \in X$ if, for every open set $U$ containing $F\left(x_{0}\right)$, there is a neighborhood $N\left(x_{0}\right)$ of $x_{0}$ in $X$ such that

$$
F(x) \subset U, \quad \forall x \in N\left(x_{0}\right) .
$$

(ii) The map $F$ is said to be lower semicontinuous at $x_{0}$ if, for every open set $U$ with $F\left(x_{0}\right) \cap U \neq \emptyset$, there is a neighborhood $N\left(x_{0}\right)$ of $x_{0}$ in $X$ such that

$$
F(x) \bigcap U \neq \emptyset, \quad \forall x \in N\left(x_{0}\right) .
$$

Lemma $2.1 \quad[10,20]$

(i) If $F$ is lower semicontinuous at $x_{0} \in X$ if and only if for any $y_{0} \in F\left(x_{0}\right)$, and any net $\left\{x_{\alpha}\right\}$ satisfying $x_{\alpha} \rightarrow x_{0}$, there exists a net $\left\{y_{\alpha}\right\}$ such that $y_{\alpha} \in F\left(x_{\alpha}\right)$ and $y_{\alpha} \rightarrow y_{0}$.

(ii) Let $F(x)$ be compact valued on $X$. Then $F$ is upper semicontinuous at $x_{0}$ if and only if for any net $\left\{x_{\alpha}\right\} \subset X$ such that $x_{\alpha} \rightarrow x_{0}$ and for every $y_{\alpha} \in F\left(x_{\alpha}\right)$, there exist $y_{0} \in F\left(x_{0}\right)$ and subset $\left\{y_{\beta}\right\}$ of $\left\{y_{\alpha}\right\}$ such that $y_{\beta} \rightarrow y_{0}$.

Definition 2.2 (Classical notion) A set-valued map $G: M \rightarrow 2^{X}$ is said to be $\ell . \alpha$-Hölder continuous at $\mu_{0}$ if there is a neighborhood $U\left(\mu_{0}\right)$ of $\mu_{0}$ such that, $\forall \mu_{1}, \mu_{2} \in U\left(\mu_{0}\right)$,

$$
G\left(\mu_{1}\right) \subseteq G\left(\mu_{2}\right)+\ell B\left(0, d^{\alpha}\left(\mu_{1}, \mu_{2}\right)\right)
$$

where $\ell \geq 0$ and $\alpha>0$. 
Definition 2.3 [2] Let $G: X \times X \rightarrow 2^{Y}$ be a set-valued map.

(i) $G$ is called h. $\beta$-Hölder strongly pseudomonotone of the first type in $E \subset X$ if, $\forall x, y \in$ $E, x \neq y$,

$$
G(x, y) \nsubseteq-i n t C \Rightarrow G(y, x)+h B\left(0, d^{\beta}(x, y)\right) \subseteq-C,
$$

where $h>0$ and $\beta>0$.

(ii) $G$ is called h. $\beta$-Hölder strongly pseudomonotone of the second type in $E \subset X$ if, $\forall x, y \in E, x \neq y$,

$$
G(x, y) \subseteq Y \backslash-\operatorname{int} C \Rightarrow G(y, x)+h B\left(0, d^{\beta}(x, y)\right) \subseteq-C,
$$

where $h>0$ and $\beta>0$.

(iii) $G$ is called quasimonotone of the first type in $E \subset X$ if, $\forall x, y \in E, x \neq y$,

$$
G(x, y) \subseteq-i n t C \Rightarrow G(y, x) \nsubseteq-i n t C
$$

(iv) $G$ is called quasimonotone of the second type in $E \subset X$ if, $\forall x, y \in E, x \neq y$,

$$
G(x, y) \nsubseteq Y \backslash-i n t C \Rightarrow G(y, x) \subseteq Y \backslash-i n t C
$$

Definition 2.4 [21] Let $(E, d)$ be a metric space and $H$ be a Hausdorff metric on the collection $\boldsymbol{C B}(E)$ of all nonempty closed bounded subsets of $E$, which is defined as

$$
H(A, B)=\max \left\{\sup _{a \in A} d(a, B), \sup _{b \in B} d(A, b)\right\}, \forall A, B \in \boldsymbol{C B}(E),
$$

where $d(a, B)=\inf _{b \in B} d(a, b)$ and $d(A, b)=\inf _{a \in A} d(a, b)$.

\section{Hölder Continuity of a Solution Map for (PGVEP1)}

In this section, we mainly discuss the Hölder continuity of a solution map for a case of the generalized vector equilibrium problem where both the set-valued map $F$ and the subset $E$ are perturbed by parameters.

Proposition 3.1 Let $F: E \times E \times M \rightarrow 2^{Y}$ and $E: \Lambda \rightarrow E$ be given. Assume that the solutions for the problem (PGVEP1) exist in a neighborhood $N(\bar{\lambda}) \times U(\bar{\mu})$ of the considered point $(\bar{\lambda}, \bar{\mu})$. Assume further that the following conditions hold: 
(i) For each $\lambda \in N(\bar{\lambda}), E(\cdot)$ is compact;

(ii) For each $\lambda \in N(\bar{\lambda}), \mu \in U(\bar{\mu})$ and $y \in E(\lambda), F(\cdot, y, \mu)$ is upper semicontinuous with compact values.

Then, for any $\lambda \in N(\bar{\lambda}), \mu \in U(\bar{\mu}), S_{1}(\lambda, \mu)$ is a compact subset in $E(\lambda)$.

Proof. It suffices to show that $S_{1}(\lambda, \mu)$ is closed in $E(\lambda)$, since $E(\lambda)$ is compact. Indeed, take any sequence $\left\{x_{n}\right\} \subset S_{1}(\lambda, \mu)$ with $x_{n} \rightarrow x_{0}$. It follows from the definition of the solutions for the problem (PGVEP1) that $\left\{x_{n}\right\} \subset E(\lambda)$ and for any $y \in E(\lambda)$, there exists $z_{n} \in F\left(x_{n}, y, \mu\right)$ satisfying

$$
z_{n} \in F\left(x_{n}, y, \mu\right) \cap Y \backslash-\operatorname{intC} \neq \emptyset, \forall n .
$$

From the compactness of $E(\lambda)$, we have $x_{0} \in E(\lambda)$. By the upper semicontinuous of $F(\cdot, y, \mu)$ and the compactness of $F\left(x_{0}, y, \mu\right)$, there exists $z_{0} \in F\left(x_{0}, y, \mu\right)$ such that $z_{n} \rightarrow$ $z_{0}$ (taking a subsequence $\left\{z_{n_{i}}\right\} \subset\left\{z_{n}\right\}$ if necessary). Noting the closedness of $Y \backslash$-intC, we have $z_{n} \rightarrow z_{0} \in Y \backslash$-intC. Then,

$$
z_{0} \in F\left(x_{0}, y, \mu\right) \cap Y \backslash-\operatorname{intC} .
$$

By the arbitrariness of $y$ and (1), we get

$$
F\left(x_{0}, y, \mu\right) \nsubseteq-\operatorname{intC}, \forall y \in E(\lambda) .
$$

Thus, $x_{0} \in S_{1}(\lambda, \mu)$ and the proof is complete.

Theorem 3.1 Assume that the solutions for the problem (PGVEP1) exist in a neighborhood $N(\bar{\lambda}) \times U(\bar{\mu})$ of the considered point $(\bar{\lambda}, \bar{\mu})$. Assume further that the following conditions hold:

(i) $E(\cdot)$ is $\ell . \alpha$-Hölder continuous at $\bar{\lambda}$ with compact values;

(ii) There are neighborhoods $N(\bar{\lambda})$ of $\bar{\lambda}$ and $U(\bar{\mu})$ of $\bar{\mu}$ and constants $h>0, \beta>0$ such that $\forall(\lambda, \mu) \in N(\bar{\lambda}) \times U(\bar{\mu}), \forall y \in \Delta(\lambda, \mu)=E(N(\bar{\lambda})) \backslash S_{1}(\lambda, \mu), \exists \widehat{x} \in S_{1}(\lambda, \mu)$ satisfying

$$
F(y, \widehat{x}, \mu)+h B\left(0, d^{\beta}(\widehat{x}, y)\right) \subseteq-C
$$

(iii) For each $\lambda \in N(\bar{\lambda})$ and each $x, y \in E(\lambda), F(x, y, \cdot)$ is $m \cdot \gamma$-Hölder continuous at $\bar{\mu}$; 
(iv) For each $\lambda \in N(\bar{\lambda})$, each $x \in E(\lambda)$ and each $\mu \in U(\bar{\mu}), F(x, \cdot, \mu)$ is $n . \delta$-Hölder continuous in $E(N(\bar{\lambda}))$.

(v) For each $\lambda \in N(\bar{\lambda}), \mu \in U(\bar{\mu})$ and $y \in E(\lambda), F(\cdot, y, \mu)$ is upper semicontinuous with compact values.

Then, the solution map $S_{1}(\cdot, \cdot)$ satisfies the following Hölder continuous condition, for any $\left(\lambda_{1}, \mu_{1}\right)$ and $\left(\lambda_{2}, \mu_{2}\right)$ in a neighborhood of $(\bar{\lambda}, \bar{\mu})$ :

$$
H\left(S_{1}\left(\lambda_{1}, \mu_{1}\right), S_{1}\left(\lambda_{2}, \mu_{2}\right)\right) \leq\left(\frac{m}{h}\right)^{\frac{1}{\beta}} d^{\frac{\gamma}{\beta}}\left(\mu_{1}, \mu_{2}\right)+\left(\frac{\ell^{\delta} n}{h}\right)^{\frac{1}{\beta}} d^{\frac{\alpha \delta}{\beta}}\left(\lambda_{1}, \lambda_{2}\right) .
$$

Proof. By virtue of Proposition 3.1, it follows from the assumptions (i) and (v) that for each $(\lambda, \mu) \in N(\bar{\lambda}) \times U(\bar{\mu}), S_{1}(\lambda, \mu)$ is a compact subset on $E(\lambda)$. Thus, it suffices to show that the solution map $S_{1}(\cdot, \cdot)$ satisfies the Hölder continuous condition.

Let $\left(\lambda_{1}, \mu_{1}\right),\left(\lambda_{2}, \mu_{2}\right) \in N(\bar{\lambda}) \times U(\bar{\mu})$. We shall divide into three steps for proving that (2) holds.

Step 1 We prove that

$$
H\left(S_{1}\left(\lambda_{1}, \mu_{1}\right), S_{1}\left(\lambda_{1}, \mu_{2}\right)\right) \leq\left(\frac{m}{h}\right)^{\frac{1}{\beta}} d^{\frac{\gamma}{\beta}}\left(\mu_{1}, \mu_{2}\right) .
$$

Obviously, if $S_{1}\left(\lambda_{1}, \mu_{1}\right)=S_{1}\left(\lambda_{1}, \mu_{2}\right)$, we have that (3) holds. So we suppose $S_{1}\left(\lambda_{1}, \mu_{1}\right) \neq$ $S_{1}\left(\lambda_{1}, \mu_{2}\right)$. There are two cases to be considered.

Case 1. $S_{1}\left(\lambda_{1}, \mu_{1}\right) \nsubseteq S_{1}\left(\lambda_{1}, \mu_{2}\right)$ and $S_{1}\left(\lambda_{1}, \mu_{1}\right) \nsupseteq S_{1}\left(\lambda_{1}, \mu_{2}\right)$. For any $x\left(\lambda_{1}, \mu_{1}\right) \in$ $S_{1}\left(\lambda_{1}, \mu_{1}\right) \backslash S_{1}\left(\lambda_{1}, \mu_{2}\right)$, by virtue of the assumption (ii), there exists $\widehat{x}\left(\lambda_{1}, \mu_{2}\right) \in S_{1}\left(\lambda_{1}, \mu_{2}\right)$ such that

$$
F\left(x\left(\lambda_{1}, \mu_{1}\right), \widehat{x}\left(\lambda_{1}, \mu_{2}\right), \mu_{2}\right)+h B\left(0, d^{\beta}\left(x\left(\lambda_{1}, \mu_{1}\right), \widehat{x}\left(\lambda_{1}, \mu_{2}\right)\right)\right) \subseteq-C .
$$

Then, $\forall z \notin-\operatorname{int} C$,

$$
d^{\beta}\left(x\left(\lambda_{1}, \mu_{1}\right), \widehat{x}\left(\lambda_{1}, \mu_{2}\right)\right) \leq \frac{1}{h} H\left(F\left(x\left(\lambda_{1}, \mu_{1}\right), \widehat{x}\left(\lambda_{1}, \mu_{2}\right), \mu_{2}\right),\{z\}\right) .
$$

Since $x\left(\lambda_{1}, \mu_{1}\right) \in S_{1}\left(\lambda_{1}, \mu_{1}\right)$, then

$$
F\left(x\left(\lambda_{1}, \mu_{1}\right), y, \mu_{1}\right) \nsubseteq-\operatorname{int} C, \quad \forall y \in E\left(\lambda_{1}\right) .
$$

Taking $y=\widehat{x}\left(\lambda_{1}, \mu_{2}\right)$, there exists

$$
z_{0} \in F\left(x\left(\lambda_{1}, \mu_{1}\right), \widehat{x}\left(\lambda_{1}, \mu_{2}\right), \mu_{1}\right) \backslash-\operatorname{int} C .
$$


By virtue of (4), (5) and the assumption (iii), we can get

$$
\begin{aligned}
d^{\beta}\left(x\left(\lambda_{1}, \mu_{1}\right), \widehat{x}\left(\lambda_{1}, \mu_{2}\right)\right) & \leq \frac{1}{h} H\left(F\left(x\left(\lambda_{1}, \mu_{1}\right), \widehat{x}\left(\lambda_{1}, \mu_{2}\right), \mu_{2}\right),\left\{z_{0}\right\}\right) \\
& \leq \frac{1}{h} H\left(F\left(x\left(\lambda_{1}, \mu_{1}\right), \widehat{x}\left(\lambda_{1}, \mu_{2}\right), \mu_{2}\right), F\left(x\left(\lambda_{1}, \mu_{1}\right), \widehat{x}\left(\lambda_{1}, \mu_{2}\right), \mu_{1}\right)\right) \\
& \leq \frac{m}{h} d^{\gamma}\left(\mu_{1}, \mu_{2}\right) .
\end{aligned}
$$

Noting the arbitrariness of $x\left(\lambda_{1}, \mu_{1}\right)$, one has

$$
\begin{aligned}
\sup _{x\left(\lambda_{1}, \mu_{1}\right) \in S_{1}\left(\lambda_{1}, \mu_{1}\right) \backslash S_{1}\left(\lambda_{1}, \mu_{2}\right)} \inf _{x\left(\lambda_{1}, \mu_{2}\right) \in S_{1}\left(\lambda_{1}, \mu_{2}\right)} d\left(x\left(\lambda_{1}, \mu_{1}\right), x\left(\lambda_{1}, \mu_{2}\right)\right) & \leq d\left(x\left(\lambda_{1}, \mu_{1}\right), \widehat{x}\left(\lambda_{1}, \mu_{2}\right)\right) \\
& \leq\left(\frac{m}{h}\right)^{\frac{1}{\beta}} d^{\frac{\gamma}{\beta}}\left(\mu_{1}, \mu_{2}\right) .
\end{aligned}
$$

Then, by the definition of metric $d(\cdot, \cdot)$, we have

$$
\begin{aligned}
\sup _{x\left(\lambda_{1}, \mu_{2}\right) \in S_{1}\left(\lambda_{1}, \mu_{2}\right)} d\left(x\left(\lambda_{1}, \mu_{1}\right), S_{1}\left(\lambda_{1}, \mu_{2}\right)\right) & =\sup _{x\left(\lambda_{1}, \mu_{1}\right) \in S_{1}\left(\lambda_{1}, \mu_{1}\right) \backslash S_{1}\left(\lambda_{1}, \mu_{2}\right)} d\left(x\left(\lambda_{1}, \mu_{1}\right), S_{1}\left(\lambda_{1}, \mu_{2}\right)\right) \\
& \leq\left(\frac{m}{h}\right)^{\frac{1}{\beta}} d^{\frac{\gamma}{\beta}}\left(\mu_{1}, \mu_{2}\right) .
\end{aligned}
$$

Using the same method of proof, we also have

$$
\sup _{x\left(\lambda_{1}, \mu_{2}\right) \in S_{1}\left(\lambda_{1}, \mu_{2}\right)} d\left(S_{1}\left(\lambda_{1}, \mu_{1}\right), x\left(\lambda_{1}, \mu_{2}\right)\right) \leq\left(\frac{m}{h}\right)^{\frac{1}{\beta}} d^{\frac{\gamma}{\beta}}\left(\mu_{1}, \mu_{2}\right) .
$$

Combining (6) and (7), we have that (3) holds.

Case 2. $S_{1}\left(\lambda_{1}, \mu_{1}\right) \subset S_{1}\left(\lambda_{1}, \mu_{2}\right)$ or $S_{1}\left(\lambda_{1}, \mu_{1}\right) \supset S_{1}\left(\lambda_{1}, \mu_{2}\right)$. Without loss of generality, we assume that $S_{1}\left(\lambda_{1}, \mu_{1}\right) \subset S_{1}\left(\lambda_{1}, \mu_{2}\right)$. From the definition of Hausdorff distance, one has

$$
\sup _{x\left(\lambda_{1}, \mu_{1}\right) \in S_{1}\left(\lambda_{1}, \mu_{1}\right)} d\left(x\left(\lambda_{1}, \mu_{1}\right), S_{1}\left(\lambda_{1}, \mu_{2}\right)\right)=0 .
$$

By using the same argument of Case 1 , we have

$$
\sup _{x\left(\lambda_{1}, \mu_{2}\right) \in S_{1}\left(\lambda_{1}, \mu_{2}\right)} d\left(S_{1}\left(\lambda_{1}, \mu_{1}\right), x\left(\lambda_{1}, \mu_{2}\right)\right) \leq\left(\frac{m}{h}\right)^{\frac{1}{\beta}} d^{\frac{\gamma}{\beta}}\left(\mu_{1}, \mu_{2}\right) .
$$

Consequently, it follows from (8) and (9) that (3) also holds.

In a word, by Cases 1 and 2, we have that (3) holds.

Step 2 Now we show that

$$
H\left(S_{1}\left(\lambda_{1}, \mu_{2}\right), S_{1}\left(\lambda_{2}, \mu_{2}\right)\right) \leq\left(\frac{\ell^{\delta} n}{h}\right)^{\frac{1}{\beta}} d^{\frac{\alpha \delta}{\beta}}\left(\lambda_{1}, \lambda_{2}\right) .
$$


Obviously, we only need to prove that (10) holds when $S_{1}\left(\lambda_{1}, \mu_{1}\right) \neq S_{1}\left(\lambda_{1}, \mu_{2}\right)$. To be similar to Step 1, we also consider two cases.

Case 1. $S_{1}\left(\lambda_{1}, \mu_{2}\right) \nsubseteq S_{1}\left(\lambda_{2}, \mu_{2}\right)$ and $S_{1}\left(\lambda_{1}, \mu_{2}\right) \nsupseteq S_{1}\left(\lambda_{2}, \mu_{2}\right)$. For any $x\left(\lambda_{2}, \mu_{2}\right) \in$ $S_{1}\left(\lambda_{2}, \mu_{2}\right) \backslash S_{1}\left(\lambda_{1}, \mu_{2}\right)$, by virtue of the assumption (ii), there exists $\widehat{x}\left(\lambda_{1}, \mu_{2}\right) \in S_{1}\left(\lambda_{1}, \mu_{2}\right)$ such that

$$
F\left(x\left(\lambda_{2}, \mu_{2}\right), \widehat{x}\left(\lambda_{1}, \mu_{2}\right), \mu_{2}\right)+h B\left(0, d^{\beta}\left(\widehat{x}\left(\lambda_{1}, \mu_{2}\right), x\left(\lambda_{2}, \mu_{2}\right)\right)\right) \subseteq-C .
$$

Then, $\forall z \notin-\operatorname{int} C$,

$$
d^{\beta}\left(\widehat{x}\left(\lambda_{1}, \mu_{2}\right), x\left(\lambda_{2}, \mu_{2}\right)\right) \leq \frac{1}{h} H\left(F\left(x\left(\lambda_{2}, \mu_{2}\right), \widehat{x}\left(\lambda_{1}, \mu_{2}\right), \mu_{2}\right),\{z\}\right) .
$$

By virtue of the assumption (i), there is $\bar{x} \in E\left(\lambda_{2}\right)$ such that

$$
d\left(\widehat{x}\left(\lambda_{1}, \mu_{2}\right), \bar{x}\right) \leq \ell d^{\alpha}\left(\lambda_{1}, \lambda_{2}\right) .
$$

(We can assume that $N(\bar{\lambda})$ is contained in the neighborhood where $E(\cdot)$ is $\ell . \alpha$-Hölder continuous.) By $x\left(\lambda_{2}, \mu_{2}\right) \in S_{1}\left(\lambda_{2}, \mu_{2}\right)$, there exists $\bar{z} \in F\left(x\left(\lambda_{2}, \mu_{2}\right), \bar{x}, \mu_{2}\right) \backslash-\operatorname{int} C$. Consequently, (11), (12) and the assumption (iv) together imply that

$$
\begin{aligned}
d^{\beta}\left(\widehat{x}\left(\lambda_{1}, \mu_{2}\right), x\left(\lambda_{2}, \mu_{2}\right)\right) & \leq \frac{1}{h} H\left(F\left(x\left(\lambda_{2}, \mu_{2}\right), \widehat{x}\left(\lambda_{1}, \mu_{2}\right), \mu_{2}\right), F\left(x\left(\lambda_{2}, \mu_{2}\right), \bar{x}, \mu_{2}\right)\right) \\
& \leq \frac{n}{h} d^{\delta}\left(\widehat{x}\left(\lambda_{1}, \mu_{2}\right), \bar{x}\right) \\
& \leq \frac{n}{h} \ell^{\delta} d^{\alpha \delta}\left(\lambda_{1}, \lambda_{2}\right) .
\end{aligned}
$$

Since the arbitrariness of $x\left(\lambda_{2}, \mu_{2}\right)$, by the definition of $d(\cdot, \cdot)$, one has

$$
\sup _{x\left(\lambda_{2}, \mu_{2}\right) \in S_{1}\left(\lambda_{2}, \mu_{2}\right)} d\left(S_{1}\left(\lambda_{1}, \mu_{2}\right), x\left(\lambda_{2}, \mu_{2}\right)\right) \leq\left(\frac{\ell^{\delta} n}{h}\right)^{\frac{1}{\beta}} d^{\frac{\alpha \delta}{\beta}}\left(\lambda_{1}, \lambda_{2}\right) .
$$

Using the same argument, we can also get

$$
\sup _{x\left(\lambda_{1}, \mu_{2}\right) \in S_{1}\left(\lambda_{1}, \mu_{2}\right)} d\left(x\left(\lambda_{1}, \mu_{2}\right), S_{1}\left(\lambda_{2}, \mu_{2}\right)\right) \leq\left(\frac{\ell^{\delta} n}{h}\right)^{\frac{1}{\beta}} d^{\frac{\alpha \delta}{\beta}}\left(\lambda_{1}, \lambda_{2}\right) .
$$

Then, we have that (10) holds.

Case 2. $S_{1}\left(\lambda_{1}, \mu_{2}\right) \subset S_{1}\left(\lambda_{2}, \mu_{2}\right)$ or $S_{1}\left(\lambda_{1}, \mu_{2}\right) \supset S_{1}\left(\lambda_{2}, \mu_{2}\right)$. It follows from the proof of Step 1 that (10) also holds.

Thus, by Cases 1 and 2 of Step 2, we have (10) holds.

Step 3 Finally, since

$$
H\left(S_{1}\left(\lambda_{1}, \mu_{1}\right), S_{1}\left(\lambda_{2}, \mu_{2}\right)\right) \leq H\left(S_{1}\left(\lambda_{1}, \mu_{1}\right), S_{1}\left(\lambda_{1}, \mu_{2}\right)\right)+H\left(S_{1}\left(\lambda_{1}, \mu_{2}\right), S_{1}\left(\lambda_{2}, \mu_{2}\right)\right),
$$

it follows from (3) and (10) that (2) holds. Therefore, the proof is complete. 
Remark 3.1 It is well known that the solution map for (PGVEP1) is, in general, not a single-valued one. Theorem 3.1 discusses Hölder continuity under the case that the solution map is a general set-valued one for (PGVEP1). Since the assumption (ii) of Theorem 3.1 is weaker than the assumption (ii) of Theorem 2.1 of [2], Theorem 3.1 is different from Theorem 2.1 of [2]. The following example shows the case, where Theorem 3.1 is applicable, but Theorem 2.1 in [2] is not applicable.

Example 3.1 Let $X=Y=R, \Lambda=M=[0,1], C=R_{+}, E(\lambda)=\left[\lambda^{2}, 1\right]$ and $F(x, y, \lambda)=$ $[-10,(y+1)(x-\lambda)]$. Direct computations show that

$$
E(\Lambda)=[0,1], \quad S_{1}(\lambda)=[\lambda, 1], \quad \forall \lambda \in \Lambda,
$$

and

$$
\Delta(\lambda)=E(\Lambda) \backslash S_{1}(\lambda)=[0, \lambda), \quad \forall \lambda \in \Lambda .
$$

Obviously, for all $\lambda \in \Lambda, E(\lambda)$ is 2.1-Hölder continuous with compact values; for all $x, y \in E(\Lambda), F(x, y, \cdot)$ is 2.1-Hölder continuous at any $\bar{\lambda} \in \Lambda$; for all $x \in E(\lambda)$ and $\lambda \in \Lambda$, $F(x, \cdot, \lambda)$ is 1.1-Hölder continuous; for all $\lambda \in \Lambda$ and $y \in E(\lambda), F(\cdot, y, \lambda)$ is continuous with compact values. Take $h=1$ and $\beta=1$. For any $\lambda \in \Lambda$ and $\forall y \in \Delta(\lambda)=[0, \lambda)$, we take $\widehat{x}=\lambda \in S_{1}(\lambda)$. Then, we have

$$
\begin{aligned}
F(y, \bar{x}, \lambda)+h B\left(0, d^{\beta}(\widehat{x}, y)\right) & =[-10,(\lambda+1)(y-\lambda)]+B(0,|\lambda-y|) \\
& =[-10,(\lambda+1)(y-\lambda)]+B(0, \lambda-y) \\
& =[-10+y-\lambda, \lambda(y-\lambda)] \subseteq-C .
\end{aligned}
$$

Hence, all assumptions of Theorem 3.1 hold and it is valid.

However, let $x=1, y=0$ and $\lambda=0$. It is not hard to see that $F(1,0,0)=[-10,1] \nsubseteq$ -intC. But for any $h>0$,

$$
F(0,1,0)+h B(0,1)=[-10,0]+h B(0,1)=[-10-h, h] \nsubseteq-C,
$$

which implies that $F(\cdot, \cdot, 0)$ is not strongly h. $\beta$-Hölder pseudomonotone of the first type in $E(\Lambda)$. And take $x=0, \lambda=1$, and $y=\frac{1}{2}$. Then $F\left(0, \frac{1}{2}, 1\right)=\left[-10,-\frac{3}{2}\right]$, but $F\left(\frac{1}{2}, 0,1\right)=$ $\left[-10,-\frac{1}{2}\right]$, which implies that $F(\cdot, \cdot, 1)$ is not quasimonotone of first type in $E(\Lambda)$. Therefore, the assumptions of Theorem 2.1 in [2] do not hold. Thus, Theorem 2.1 in [2] is not applicable.

Remark 3.2 If the subset $E \subseteq X$ is fixed, then $S_{1}(\lambda, \mu)$ reduces to $S_{1}(\mu)$ to (PGVEP1). Thus the Hölder continuous condition of $S_{1}(\cdot)$ can be obtained by applying Theorem 3.1 directly. 


\section{Hölder Continuity of Solution Map for (PGVEP2)}

In this section, we discuss the Hölder Continuity of a solution map for another case of the generalized vector equilibrium problem where both the set-valued map $F$ and the subset $E$ are perturbed by parameters. We first prove the following result.

Proposition 4.1 Let $F: E \times E \times M \rightarrow 2^{Y}$ and $E: \Lambda \rightarrow E$ be given. Assume that the solutions for the problem (PGVEP2) exist in a neighborhood $N(\bar{\lambda}) \times U(\bar{\mu})$ of the considered point $(\bar{\lambda}, \bar{\mu})$. Assume further that the following conditions hold:

(i) For each $\lambda \in N(\bar{\lambda}), E(\cdot)$ is compact;

(ii) For each $\lambda \in N(\bar{\lambda}), \mu \in U(\bar{\lambda})$ and $y \in E(\lambda), F(\cdot, y, \mu)$ is lower semicontinuous.

Then, for any $\lambda \in N(\bar{\lambda}), S_{2}(\lambda, \mu)$ is a compact subset in $E(\lambda)$.

Proof. Since $E(\lambda)$ is compact, it suffices to show that $S_{2}(\lambda, \mu)$ is closed in $E(\lambda)$. Indeed, taking any sequence $\left\{x_{n}\right\} \subset S_{2}(\lambda, \mu)$ satisfying $x_{n} \rightarrow x_{0}$, we need to show that $x_{0} \in$ $S(\lambda, \mu)$. Since $x_{n} \in E(\lambda)$ and $E(\lambda)$ is compact, we have $x_{0} \in E(\lambda)$. For each $y \in E(\lambda)$ and $z_{0} \in F\left(x_{0}, y, \mu\right)$, by assumption (ii), there exists $z_{n} \in F\left(x_{n}, y, \mu\right)$ such that $z_{n} \rightarrow z_{0}$. Since $Y \backslash$-intC is closed and $F\left(x_{n}, y, \mu\right) \subseteq Y \backslash$-intC, we get

$$
z_{0} \in Y \backslash-\operatorname{intC}
$$

It follows from (13) and the arbitrariness of $y$ and $z_{0}$ that

$$
F\left(x_{0}, y, \mu\right) \subseteq Y \backslash-\operatorname{intC}, \forall y \in E(\lambda) .
$$

Thus, $z_{0} \in S_{2}(\lambda, \mu)$ and the proof is complete.

Theorem 4.1 Assume that the solutions for the problem (PGVEP2) exist in a neighborhood $N(\bar{\lambda}) \times U(\bar{\mu})$ of the considered point $(\bar{\lambda}, \bar{\mu})$. Assume further that (i), (iii) and (iv) in Theorem 3.1 hold and (ii) and (v) are, respectively, replaced by

(ii') There are neighborhoods $N(\bar{\lambda})$ of $\bar{\lambda}$ and $U(\bar{\mu})$ of $\bar{\mu}$ and constants $h>0, \beta>0$ such that $\forall(\lambda, \mu) \in N(\bar{\lambda}) \times U(\bar{\mu}), \forall y \in \Delta(\lambda, \mu)=E(N(\bar{\lambda})) \backslash S_{2}(\lambda, \mu), \exists \widehat{x} \in S_{2}(\lambda, \mu)$ satisfying

$$
F(y, \widehat{x}, \mu)+h B\left(0, d^{\beta}(\widehat{x}, y)\right) \subseteq-C ;
$$


(v') For each $\lambda \in N(\bar{\lambda}), \mu \in U(\bar{\mu})$ and $y \in E(\lambda), F(\cdot, y, \mu)$ is lower semicontinuous.

Then, the solution map $S_{2}(\cdot, \cdot)$ satisfies the following Hölder continuous condition, for any $\left(\lambda_{1}, \mu_{1}\right)$ and $\left(\lambda_{2}, \mu_{2}\right)$ in a neighborhood of $(\bar{\lambda}, \bar{\mu})$ :

$$
H\left(S_{2}\left(\lambda_{1}, \mu_{1}\right), S_{2}\left(\lambda_{2}, \mu_{2}\right)\right) \leq\left(\frac{m}{h}\right)^{\frac{1}{\beta}} d^{\frac{\gamma}{\beta}}\left(\mu_{1}, \mu_{2}\right)+\left(\frac{\ell^{\delta} n}{h}\right)^{\frac{1}{\beta}} d^{\frac{\alpha \delta}{\beta}}\left(\lambda_{1}, \lambda_{2}\right) .
$$

Proof. For any $(\lambda, \mu) \in N(\bar{\lambda}) \times U(\bar{\mu})$, by the definitions of the solutions for the problems (PGVEP1) and (PGVEP2), we know $S_{2}(\lambda, \mu) \subseteq S_{1}(\lambda, \mu)$. By the similar method of Theorem 3.1, the solution map $S_{2}(\cdot, \cdot)$ satisfies the Hölder continuous condition. This completes the proof.

Now we give an example to illustrate that (ii') in Theorem 4.1 is essential.

Example 4.1 Let $X, Y, C$ be given as in Example 3.1. Let $\Lambda \equiv M=[1,2], E(\lambda)=$ $[\lambda-1,1]$ and $F(x, y, \lambda)=[\lambda x(x-y),+\infty)$.

Obviously, for all $\lambda \in \Lambda, E(\lambda)$ is 1.1-Hölder continuous with compact values; for all $x, y \in E(\Lambda), F(x, y, \cdot)$ is 1.1-Hölder continuous at any $\bar{\lambda} \in \Lambda ;$ for all $x \in E(\lambda)$ and $\lambda \in \Lambda$, $F(x, \cdot, \lambda)$ is 2.1-Hölder continuous; for all $\lambda \in \Lambda$ and $y \in E(\lambda), F(\cdot, y, \lambda)$ is continuous. So all assumptions of Theorem 4.1 hold except for the assumption (ii'). It follows from the direct computations that

$$
E(\Lambda)=[0,1], \quad S_{2}(1)=\{0,1\} \quad \text { and } \quad S_{2}(\lambda)=\{1\}, \forall \lambda \in(1,2] .
$$

Hence $S_{2}(\cdot)$ is even not lower semicontinuous at $\lambda=1$. The reason is that the assumption (ii') is violated. Indeed, if $\bar{x}=0$, for any $h>0$, there exists $y=\frac{1}{2} \in \Delta(1)=(0,1)$ such that

$$
\begin{aligned}
F(y, \bar{x}, 1)+h B\left(0, d^{\beta}(\widehat{x}, y)\right) & =\left[\frac{1}{2}\left(\frac{1}{2}-0\right),+\infty\right)+h B\left(0,\left|0-\frac{1}{2}\right|\right) \\
& =\left[\frac{1}{4},+\infty\right)+h B\left(0, \frac{1}{2}\right) \\
& =\left[\frac{1}{4}-\frac{h}{2},+\infty\right) \nsubseteq-C ;
\end{aligned}
$$

if $\bar{x}=1$, for any $h>0$, there exists $y=\frac{1}{2} \in \Delta(1)=(0,1)$ such that

$$
\begin{aligned}
F(y, \bar{x}, 1)+h B\left(0, d^{\beta}(\widehat{x}, y)\right) & =\left[\frac{1}{2}\left(\frac{1}{2}-1\right),+\infty\right)+h B\left(0,\left|1-\frac{1}{2}\right|\right) \\
& =\left[-\frac{1}{4},+\infty\right)+h B\left(0, \frac{1}{2}\right) \\
& =\left[-\frac{1}{4}-\frac{h}{2},+\infty\right) \not \leq-C .
\end{aligned}
$$

Therefore, (ii') is violated. 
Remark 4.1 (1) If $Y=R, C=R_{+}$and $F$ is a single-valued map, then the problems (PGVEP1) and (PGVEP2) reduce to the parametric scalar equilibrium problem $\left(E P_{\lambda, \mu}\right)$ considered in [11]. Note that the assumption (ii) in Theorem 3.1 ( or (ii) in Theorem 4.1) is the same as corresponding one of Theorem 4.2 in [11]. If the assumption (i) of Theorem 4.2 in [11] holds, i.e., $f(\cdot, \cdot, \mu)$ is strongly pseudomonotone, the assumption (ii) in Theorem 3.1 (or (ii,) in Theorem 4.1) is obviously satisfied. Since the solution set $S_{1}(\lambda, \mu)$ is single-valued as a result of the proof of in [11], the condition (v) in Theorem 3.1 ( or ( $\left.v^{\prime}\right)$ in Theorem 4.1) holds. So, Theorem 3.1 and Theorem 4.1 generalize Theorem 4.2 in [11].

(2) Furthermore, let $E(\lambda)=E$. The problems (PGVEP1) and (PGVEP2) reduce to the parametric scalar equilibrium problem $\left(E P_{\mu}\right)$ considered in [11]. Theorem 3.1 and Theorem 4.1 also generalize Proposition 3.3 in [11]. The following example shows the case, where our Theorem 3.1 and Theorem 4.1 are applicable, but Proposition 3.3 in [11] is not applicable.

Example 4.2 Let $X, Y, M, C$ be given as in Example 3.1. Let $E=[0,1]$ and $F(x, y, \mu)=$ $(y+1)\left(x-\mu^{2}\right)$. It follows from the direct computations that

$$
S_{1}(\mu)=\left[\mu^{2}, 1\right], \quad \forall \mu \in M
$$

and

$$
\Delta(\mu)=E \backslash S_{1}(\mu)=\left[0,1-\mu^{2}\right), \forall \mu \in M .
$$

It follows from Example 3.1 that all conditions of Theorem 3.1 and Theorem 4.1 are satisfied. Then, Theorem 3.1 and Theorem 4.1 are applicable. However, let $x=1, y=0$ and $\mu=0$. It is not hard to see that $F(1,0,0)=1$, and for any $h>0, F(0,1,0)=0 \not \leq$ $-h$, which implies that $F(\cdot, \cdot, 0)$ is not strongly h. $\beta$-Hölder pseudomonotone. Therefore, Proposition 3.3 in [11] is not applicable.

\section{Acknowledgment}

The authors thank two anonymous referees for valuable comments and suggestions, which helped improving the paper. 


\section{References}

[1] L.Q. Anh and P.Q. Khanh, Semicontinuity of the solution set of parametric multivalued vector quasiequilibrium problems, J. Math. Anal. Appl. 294(2004), 699-711.

[2] L.Q. Anh and P.Q. Khanh, On the Hölder continuity of solutions to multivalued vector equilibrium problems, J. Math. Anal. Appl. 321(2006), 308-315.

[3] L.Q. Anh and P.Q. Khanh, Uniqueness and Hölder continuity of solution to multivalued vector equilibrium problems in metric spaces, J. Glob. Optim. 37(2007), 449-465.

[4] L.Q. Anh and P.Q. Khanh, Vaious kinds of semicontinuity of the solution sets to symmetric multievalued vector quasiequilibium problems, J. Glob. Optim. 41(2008), 539-558.

[5] L.Q. Anh and P.Q. Khanh, Sensitivity analysis for multivalued quasiequilibrium problems in metric spaces: Hölder continuity of solutions, J. Glob. Optim. 42(2008), 515-531.

[6] L.Q. Anh and P.Q. Khanh, Semicontinuity of solution sets to parametric quasivariational inclusions with applications to traffic networks, I: Upper semicontinuities, Set-val. Anal. 16(2008), 267-279.

[7] L.Q. Anh and P.Q. Khanh, Semicontinuity of solution sets to parametric quasivariational inclusions with applications to traffic networks, II: Lower semicontinuities applications, Set-val. Anal. doi: 10.1007/sl 1228-008-0082-z.

[8] Q.H. Ansari and F.B. Fabian, Generalized Vector Quasi-Equilibrium Problems with Applications, J. Math. Anal. Appl. 277(2003), 246-256.

[9] Q.H. Ansari and J.C. Yao, An Existence Result for the Generalized Vector Equilibrium Problem, Appl. Math. Lett. 12(1999), 53-56.

[10] J. P. Aubin and I. Ekeland, Applied Nonlinear Analysis, John Wiley and Sons, New York, 1984.

[11] M. Bianchi and R. Pini, A note on stability for parametric equilibrium problems, Oper. Res. Lett. 31(2003), 445-450. 
[12] G.Y. Chen, X.Q. Yang and H. Yu, A Nonlinear Scalarization Function and Generalized Quasi-Vector Equilibrium Problems, J. Glob. Optim. 32(2005), 451-466.

[13] Y. H. Cheng and D. L. Zhu, Global stability results for the weak vecor variarional inequlality, J. Glob. Optim. 32(2005), 543-550.

[14] E. Klein and A.C. Thompson, Theory of Correspondences, Wiley, New York. (1984).

[15] S. J. Li and C. R. Chen, Stability of weak vecor variational inequality, Nonlinear Anal.(2008), doi:10.1016/j.na.2008.02.032.

[16] S. J. Li, G. Y. Chen and K. L. Teo, On the stability of generalized vector quasivariational inequlity problems, J. Optim. Theory Appl. 113(2002)283-295.

[17] S.J. Li, K.L. Teo and X.Q. Yang, Generalized vector quasi-equilibrium problems, Math. Methods Oper. Res. 61(2005), 385-397.

[18] S.J. Li, K.L. Teo and X.Q. Yang, A remark on a standard and linear vector network equilibrium problem with capacity constraints, European J. Oper. Res. 184(2008), $13-23$.

[19] M. Ait Mansour and H. Riahi, Sensitivity Analysis for Abstract Equilibrium Problems, J.Math. Anal. Appl. 306(2005), 684-691.

[20] N. X. Tan, Quasi-variational inequalities in topological linear locally convex Hausdorff spaces, Math. Nachr. 122(1985), 231-245.

[21] S.B. Nadler, Multi-valued contraction mappings, Pacific J. Math. 30(1969), 475-488.

[22] N.D. Yen, Hölder continuity of solutions to parametric variational inequalities, Appl. Math. Opim. 31(1995), 245-255. 